\title{
Oral Formulation of Paclitaxel and Erlotinib Polymeric Nanoparticles: A Potential Combination to Treat Breast Cancer
}

\author{
Tahir Khuroo $^{1 *}$, Umme Atifa ${ }^{2}$ and Zeenat Iqbal ${ }^{2 *}$ \\ ${ }^{1}$ Irma Lerma Rangel College of Pharmacy, Texas A\&M Health Science Center, Texas A\&M University, USA \\ ${ }^{2}$ Department of Pharmaceutics, SPER, Jamia Hamdard, India
}

*Corresponding author: Zeenat Iqbal, Associate Professor, Dept. of Pharmaceutics, SPER, Jamia Hamdard, New Delhi-110062, India

Tahir Khuroo, Research Scientist, College of Pharmacy, Texas A\&M University, College Station, TX-77840, USA

ARTICLE INFO

Received: 幽 October 21, 2020

Published: 幽 November 03, 2020

Citation: Tahir Khuroo, Umme Atifa, Zeenat Iqbal. Oral Formulation of Paclitaxel and Erlotinib Polymeric Nanoparticles: A Potential Combination to Treat Breast Cancer. Biomed J Sci \& Tech Res 31(4)2020. BJSTR. MS.ID.005129.

\begin{abstract}
Cancer treatment continues to remain a challenge and requires concerted efforts towards designing and development of newer forms of drug delivery systems. Concurrently, the practitioners are in a process of testing singular as well as combination of old and new APIs commensurate to the treatment challenges associated with specific type of cancer forms. Till recently, as reported, by many researchers polymeric nanoparticles were only exploited to encapsulate single drugs, but cognizance of clinical evidence for fruitful use of anticancer drug combinations, has yielded, many research reports of co-encapsulating two or more active moieties but not without formulation difficulties. Foremost, the inherent pharmacotechnical challenges of an anticancer drug invariably makes its formulation a difficult premise, additionally co-encapsualting two different drugs adds onto this challenge. Moreover the physiological limitation like susceptibility to Pgp efflux cannot be ignored. Keeping all these facts into consideration a stable nanoformulation of paclitaxel and erlotinib (PE-NPs) could be developed and sequentially optimized.
\end{abstract}

Abbreviations: TK: Tyrosine Kinase; DSB: Double-Strand Breaks; HDR: HomologyDirected Recombinational Repair; NHEJ: Nonhomologous End Joining; HDR: HomologyDirected Recombinational Repair; EGFR: Epidermal Growth Factor Receptor; EPR: Enhanced Permeation and Retention

\section{Introduction}

Cancer has long remained a difficult to treat disease. Modern diagnostics has allowed an improved detection but the lack of availability of effective drugs has remained a big challenge. Often in pursuit of getting the best suited regimen, the medical practitioners resort to creating concoctions and compositions of different drugs which are experimentally used, often with positive outcomes. This has led to a keen interest of drug delivery scientists in designing and optimizing possible drug delivery platforms for carrying high payloads of combination drugs with suitable therapeutic outcomes. Advent of nanotechnology and its progression to create "nanomedicines" has added impetus to the modern cancer chemotherapeutics. An understanding of the existing cancer chemotherapeutics and thorough recce of the available literature has revealed the suitability of paclitaxel and erlotinib as a combination drug therapy for treatment of breast cancer and an insight into the delivery platforms presented polymeric nanosystem/nanomedicine to be a suitable carrier for a combination thereof.

Rationale of Using Oral Nanoparticles of Paclitaxel and Erlotinib

\section{Why Paclitaxel?}

Historically speaking, paclitaxel continues to be a first line therapy for different types of solid tumors and has recently been 
promoted as the first line treatment for metastatic breast cancer. Paclitaxel is the only clinically-validated nanomedicine, which is actually the albumin bound nanoparticles of paclitaxel (nabPTX), approved by US-FDA as the first line chemotherapy [1]. Patients with HER2-positive solid tumors benefited from paclitaxel, regardless of estrogen-receptor status, but paclitaxel did not benefit patients with HER2-negative, estrogen-receptor-positive cancers [2]. Paclitaxel in small doses show cytostatic activity and profound anti-angiogenesis.

\section{Why Erlotinib?}

EGFR tyrosine kinase (TK) inhibitor, erlotinib represents a class of compounds that can prevent EGFR autophosphorylation and interrupt downstream signaling, including the mitogen-activated protein kinase (MAPK) and phosphatidylinositol 3-kinase/ AKT pathways, thus inhibiting tumor cell proliferation and survival [3]. The DNA damage response is commonly triggered on generation of DNA double-strand breaks (DSB), which can be repaired via two principal pathways: homology-directed recombinational repair (HDR) and nonhomologous end joining (NHEJ). There is a novel link between erlotinib, a potent EGFR inhibitor, DNA damage, and homology-directed recombinational repair (HDR) in human tumor cells. Erlotinib suppresses HDR [4]. Epidermal growth factor receptor (EGFR) is expressed in several solid tumors and is reported to be up to $91 \%$ [5]. One study has tested the strategy of combining erlotinib (EGFR tyrosine kinase inhibitor) in solid tumors of breast cancer and its metastasis [6].

\section{Why paclitaxel and erlotinib combination?}

When multiple drugs are administered separately, each drug acts according to its own distinct pharmacology. Because drugs differ in their pharmacokinetic and pharmacodynamic properties, there is no certitude that target cells or tissues will synchronously receive optimal levels of each therapeutic entity. Conversely, when drugs are combined in a single NP carrier, the spatiotemporal exposure of each drug can be controlled more precisely and this may translate to a synergistic action of the selected API(s) [7]. Both the drugs target solid tumors by the mechanisms different from each other hence, the synergism can be incurred. Combination therapy will be able to overcome resistance as well as cross resistance often associated with conventional chemotherapy of cancer. Since both the drugs produce side effects different from each other at higher doses but when used in combination, dose can be reduced and hence the side effects like bone marrow depression especially neutropenia from paclitaxel and interstitial lung disease, renal failure, hepatotoxicity with or without hepatic impairment, gastrointestinal perforation, myocardial infraction/ ischemia by erlotinib can be prevented to a large extent. Both the drugs show antiangiogenic activity by inhibiting multiple number of growth factors and preapoptotic factors in similar as well as different from each other, hence possibility of resistance would be too less as compared to single anti-angiogenic drug therapy [8].

\section{Why oral therapy?}

Cancer chemotherapy heavily relies on intravenous delivery of most dosage regimen. It is often marred with multitude of challenges which include severe side effects of the drugs consumed and overt financial liability which often leads to detrimental decision of patient non-compliance, ultimately compromising the therapeutic outcomes. It is noted that I.V infusion, may lead to higher rate of complications such as thrombosis and infections which are reportedly about $20-60 \%$ with chronic venous access devices [9] which are commonly used for chemotherapeutics. These factors add onto the treatment burden and patients often elicit preference for oral chemotherapy. Henceforth an oral delivery system would be highly acceptable to patients and this generates a demand for exploration of a viable oral system. Bioavailability of erlotinib is erratic and varies from $60-100 \%$. Erlotinib administered in presence of proton pump inhibitors reduces its bioavailability and Cmax by $46 \%$ and $61 \%$ respectively and if co-administered with ranitidine reduces bioavailability and $\mathrm{Cmax}$ by $33 \%$ and $54 \%$ respectively. Similarly, bioavailability of paclitaxel is only $6.5 \%$, however some authors claim that it varies from $13-31 \%$, hence, by encapsulating these drugs into nanoparticles, it can help to maintain and increase their bioavailability [10].

\section{Why polymeric nanoparticles?}

Nanoparticles of various polymers are biocompatible, biodegradable and non-toxic. Polymeric nanoparticles have much larger surface area to volume ratio for bioavailability enhancement. They have the potential to cause reversion of multidrug resistance by P-gp because of small size of nanoparticles which can bypass the P-gp and also by using the surfactants like PVA and tween 80, polymeric nanoparticles block P-gp receptor until drug gets concentrated inside. Because of small size, nanoparticles have got the scope to penetrate in tumor cells and also cross BBB in case solid tumor is in brain or if metastasized in brain. Polymeric nanoparticles have shown controlled drug delivery at the tumor site and have also been used in radio labeling and cancer imaging. They have also got the ability to by passes RES and phagocytosis by macrophages and by using different ligands on the surface of polymer of nanoparticles drug targeting on a particular tumor can be achieved. Nanoparticles have been found to show enhanced permeation and retention (EPR) of the encapsulated drug [11,12].

\section{Hypothesis}

Most anticancer drugs are associated with numerous pharmacotechnical challenges, which limit their clinical potential and often require pharmaceutical interventions in terms of designing and optimizing delivery systems. The two selected drugs, like most anticancer moieties, exhibit low oral bioavailability which may be due to poor solubility, low GIT permeability, efflux by P-glycoproteins like ABC transporters, hepatic first pass metabolism or the local side effects to gastrointestinal tract 
due to drug exposure. These limitations often preclude oral consumption of anticancer drugs and necessitates a switch over to parenteral route or explore better oral drug delivery system(s), which can circumvent these caveats. It is henceforth hypothesized that a logically designed PLGA polymeric nanoparticulate drug delivery system could be explored for carrying high payloads of encapsulated antineoplastic agents, preventing their exposure to the hostile gastrointestinal environment. The prudently optimized dual drug loaded nano-sized polymeric particles could successfully evade recognition by reticuloendothelial system and also escape identification by P-glycoproteins and cytochrome P450 systems and increase their circulation time.

Additionally, the purported polymeric nanoparticles owing to their small size, use of different surfactants and co-surfactants would eventually improve their cellular uptake and transport, eliciting an improved pharmacokinetic and pharmacodynamic (PK) PD) profiles. Based on the rationale discussed and the formulated hypothesis, the current research majorly proposes its major objective as: "Design, development, optimization and evaluation of nanopotentiated polymeric carrier for co-delivery of erlotinib and paclitaxel for breast cancer treatment."

\section{Conclusion}

In conclusion, the nanopotentiation of paclitaxel and erlotinib via suitably designed polymeric oral drug delivery system (PE-NPs) offers unique advantages of spatiotemporal targeting, controlled drug release and PgP inhibition and is proposed as a highly patient compliant breast cancer treatment approach. The use of dual drug delivery and its circumvention of drug resistance by Pgp inhibition along with reduction in the anticipated side effects seem promising but require further substantiation following which the system will see both preclinical as well as clinical usage. Additionally, surface modification of the particles with suitable ligands or moieties like antibodies will enhance its targetability and assure its site specific delivery with reduced dose, dosing frequency and a significant reduction in devastating side effects which are often associated with anticancer chemotherapeutics.

ISSN: 2574-1241

DOI: $10.26717 / B J S T R .2020 .31 .005129$

Tahir Khuroo, Zeenat Iqbal. Biomed J Sci \& Tech Res

(C) (i) This work is licensed under Creative

Submission Link: https://biomedres.us/submit-manuscript.php

\section{References}

1. Bertrand N, J Wu, X Xu, N Kamaly, OC Farokhzad (2014) Cancer nanotechnology: the impact of passive and active targeting in the era of modern cancer biology. Adv Drug Deliv Rev 66: 2-25.

2. Hayes Daniel F, Ann D Thor, Lynn G Dressler, Donald Weaver, Susan Edgerton, et al. (2007) HER2 and Response to Paclitaxel in Node-Positive Breast Cancer. New England Journal of Medicine 357(15): 1496-1506.

3. Tan Antoinette R, Xiaowei Yang, Stephen M Hewitt, Arlene Berman, Erin R Lepper, et al. (2004) Evaluation of Biologic End Points and Pharmacokinetics in Patients with Metastatic Breast Cancer After Treatment with Erlotinib, an Epidermal Growth Factor Receptor Tyrosine Kinase Inhibitor. Journal of Clinical Oncology 22(15): 30803090 .

4. Li L, H Wang, ES Yang, CL Arteaga, F Xia (2008) Erlotinib Attenuates Homologous Recombinational Repair of Chromosomal Breaks in Human Breast Cancer Cells. Cancer Research 68(22): 9141-9146.

5. Schneider Bryan P, Kathy D Miller (2005) Angiogenesis of Breast Cancer Journal of Clinical Oncology 23(8): 1782-1790.

6. Specht JM, VK Gadi, JR Gralow, LA Korde, HM Linden, et al. (2017) Abstract P4-22-11: Combined targeted therapies for advanced triple negative breast cancer: A phase II trial of nab-paclitaxel and bevacizumab followed by maintenance targeted therapy with bevacizumab and erlotinib. Cancer Research 77: 4-22.

7. Hartkamp A (2000) Totally implantable venous access devices: evaluation of complications and a prospective comparative study of two different port systems. The Netherlands Journal of Medicine 57(6): 215223.

8. Schwarz Roderich E, Daniel G Coit, Jeffrey S Groeger (2000) Transcutaneously Tunneled Central Venous Lines in Cancer Patients: An Analysis of Device-Related Morbidity Factors Based on Prospective Data Collection. Annals of Surgical Oncology 7(6): 441-449.

9. Hilton JF, Tu D, Seymour L, Shepherd FA, Bradbury PA (2013) An evaluation of the possible interaction of gastric acid suppressing medication and the EGFR tyrosine kinase inhibitor erlotinib. Lung cancer 82(1): 136-142.

10. Prasad R, Pandey R, Varma A, Barman I (2016) Polymer based nanoparticles for drug delivery systems and cancer therapeutics. Natural polymers for drug delivery. CABI Oxfordshire: 53-70.

11. Guterres SS, Paese K, Pohlmann AR (2019) Polymeric Nanoparticles. In Nanocosmetics: 73-94.

12. Khuroo T, Verma D, Talegaonkar S, Padhi S, Panda AK (2014) Topotecantamoxifen duple PLGA polymeric nanoparticles: investigation of in vitro, in vivo and cellular uptake potential. International journal of pharmaceutics 473(1-2): 384-394.

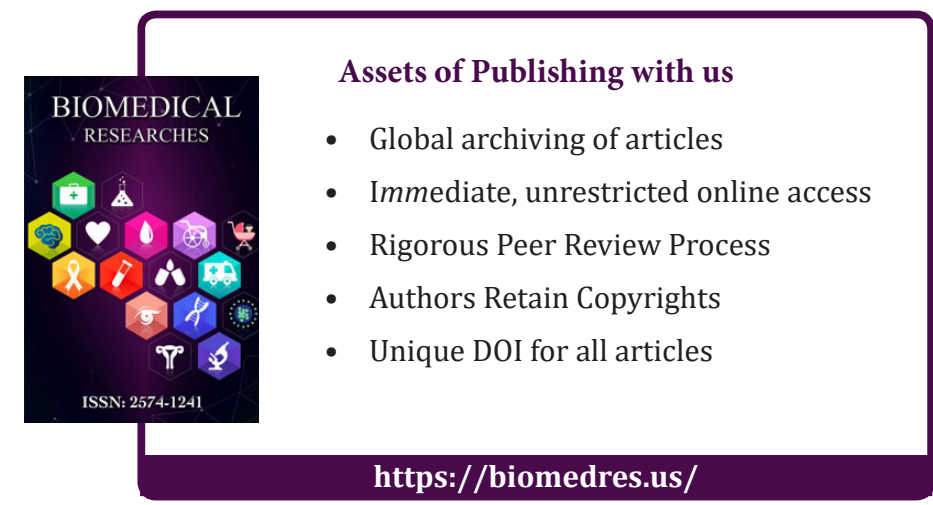

Copyright@ Tahir Khuroo, Zeenat Iqbal | Biomed J Sci \& Tech Res | BJSTR. MS.ID.005129. 